\title{
Quantitative Ultrasound Assessment of the Rat Cervix
}

\author{
Barbara L. McFarlin, PhD, CNM, RDMS, \\ William D. O'Brien, Jr, PhD, Michael L. Oelze, PhD, \\ James F. Zachary, DVM, PhD, Rosemary C. White-Traut, DNSC
}

\begin{abstract}
Objective. The purpose of this research was to detect cervical ripening with a new quantitative ultrasound technique. Methods. Cervices of 13 nonpregnant and 65 timed pregnant (days 15, 17, 19, 20, and 21 of pregnancy) Sprague Dawley rats were scanned ex vivo with a 70-MHz ultrasound transducer. Ultrasound scatterer property estimates (scatterer diameter [SD], acoustic concentration [AC], and scatterer strength factor [SSF]) from the cervices were quantified and then compared to hydroxyproline and water content. Insertion loss (attenuation) was measured in 3 rats in each of the 6 groups. Discriminant analysis was used to predict gestational age group (cervical ripening) from the ultrasound variables SD, SSF, and AC. Results. Differences were observed between the groups (SD, AC, and SSF; $P<.0001)$. Quantitative ultrasound measures changed as the cervix ripened: (1) SD increased from days 15 to 21; (2) AC decreased from days 15 to 21; and (3) SSF was the greatest in the nonpregnant group and the least in the day 21 group. Cervix hydroxyproline content increased as pregnancy progressed $(P<.003)$ and correlated with group, SD, AC, and SSF $(P<.001)$. Discriminant analysis of ultrasound variables predicted $56.4 \%$ of gestational group assignment $(P<.001)$ and increased to $77 \%$ within 2 days of the predicted analysis. Cervix insertion loss was greatest for the nonpregnant group and least for the day 21 group. Conclusions. Quantitative ultrasound predicted cervical ripening in the rat cervix, but before use in humans, quantitative ultrasound will need to predict gestational age in the later days of gestation with more precision. Key words: cervical attenuation; cervical ripening; collagen; preterm birth; quantitative ultrasound; rat cervix.
\end{abstract}

\begin{abstract}
Abbreviations
AC, acoustic concentration; GA, gestational age; QUS, quantitative ultrasound; $\mathrm{RF}$, radio frequency; $\mathrm{ROI}$, region of interest; SD, scatterer diameter; SSF, scatterer strength factor
\end{abstract}

Received March 6, 2006, from the Department of Maternal Child Nursing, University of Illinois at Chicago, Chicago, Illinois USA (B.L.M., R.C.W.-T.); and Bioacoustics Research Laboratory, Department of Electrical and Computer Engineering (W.D.O., M.L.O.), and Department of Pathobiology (J.F.Z.), University of Illinois at Urbana-Champaign, Urbana, Illinois USA. Revision requested March 30, 2006. Revised manuscript accepted for publication April 10, 2006.

This work was supported by the American Institute of Ultrasound in Medicine Endowment for Education and Research, National Institutes of Health grant F31 NR08191-01A1, a March of Dimes nursing scholarship, and an Illinois Veterans grant.

Address correspondence to Barbara L. McFarlin, PhD, CNM, RDMS, University of Illinois at Chicago, M/C 802, Room 858, 845 S Damen Ave, Chicago, IL 60612 USA.

E-mail: bmcfar1@uic.edu

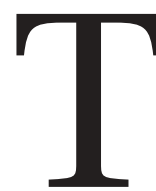

he underlying mechanisms preceding labor are not yet fully understood. Preterm birth is the second leading cause of infant mortality in the United States. ${ }^{1}$ Among the survivors, $20 \%$ to $25 \%$ have a major disability, ${ }^{2}$ and it is estimated that just the initial neonatal care costs $\$ 6$ billion annually. ${ }^{3}$ The preterm birth rate was $12.5 \%$ in 2004 , and the $16 \%$ increase in the incidence of preterm birth in the United States between 1990 and 2004 is an indication of the seriousness of this problem. ${ }^{4}$ Reducing the preterm birth rate to $7 \%$ in the United States is a goal of the Healthy People 2010 initiative. ${ }^{5}$

As early as 1947, Danforth ${ }^{6}$ described the corpus of the uterus (mainly smooth muscle and contractile) and the cervix (mainly connective tissue and noncontractile) as histologic and functionally different structures. Interventions to delay preterm birth have focused on modifying the final phase of extensive uterine contractions (smooth muscle) and have not addressed the phasic nature of labor, which involves a long period of cervical ripening before the active phase of extensive uterine contractions and cervical 
dilatation. Without a ripe cervix, uterine contractions alone cannot accomplish the work of labor and birth. ${ }^{7-9}$ Clinical and research efforts to modify cervical ripening have been limited by the lack of a reliable, valid, and noninvasive technique to measure cervical ripening. This research tested a new application of a noninvasive ultrasound technique, quantitative ultrasound (QUS), to determine cervical ripening in the rat.

Conventional B-mode ultrasonographic images are derived from backscattered radio frequency (RF) echo signals. ${ }^{10}$ The RF echoes contain frequency-dependent information about the smallerscale structures (less than the wavelength of sound) in the tissues. ${ }^{11}$ B-mode processing removes the frequency-dependent information. To quantify smaller-scale structures in tissues, the frequency-dependent information must be used. ${ }^{11,12}$

Scatterer property estimates related to tissue microstructure can be made from the ultrasound backscatter RF signals. ${ }^{13}$ When an acoustic wave propagates through tissues, the spatially varying impedance differences cause acoustic waves to be scattered. ${ }^{14}$ Acoustic scattering theories for biological tissues assume that the tissues can be modeled as either discretely or continuously varying distributions of mass density and bulk compressibility. ${ }^{15}$ Scattering models from simple shapes (spheres and cylinders) have been solved analytically. ${ }^{16}$ To estimate 3 unique tissuebased parameters (scatterer diameter [SD], scatterer acoustic concentration $[\mathrm{AC}]$, and scatterer strength factor [SSF]), it is necessary to assume a geometric model (called a form factor) for the scatterers. These parameters were estimated by modeling the tissues as scatterers with a Gaussian form factor. The Gaussian form factor assumes that the scatterers do not have sharp discontinuities in acoustic impedance with the surrounding tissues (like glass beads in agar) but have more of a gradual change in impedance with surrounding tissues. The Gaussian form factor model was theorized to be appropriate for cervix tissue because this form factor has been shown to be a good predictor of scattering in many soft tissues. ${ }^{17-21}$ The Gaussian form factor was chosen from several other available form factors because it gave the smallest $\chi^{2}$ value relative to the scattered spectra. Furthermore, the properties of the spherical Gaussian model allowed rapid processing because it could be linearized in log space. ${ }^{12}$

The scatterer property estimates are made by comparing the backscatter power spectrum from a region of interest $(\mathrm{ROI})$ in the tissues being evaluated, to a theoretical backscattered power spectrum. The backscattered power spectrum is parameterized to yield 3 quantifiable tissuebased parameters: SD, AC, and SSF. Scatterer diameter is related to the correlational length of the underlying structure. Scatterer AC is the number of scatterers per unit volume times the square of the relative impedance difference between scatterer and background. Scatterer strength factor is a combination of scattering size and scatterer AC. A further explanation of calculating the scatterer property estimates can be found in previous publications. . $^{12,22,23}$

The cause of scattering has been hypothesized to be related to the amount of collagen in the tissues ${ }^{24-26}$ and to the structure and function of the extracellular matrix. ${ }^{27}$ The cervix is mainly composed of collagen ( $70 \%$ type I collagen, $30 \%$ type III collagen, and a small amount of type IV collagen) and only $5 \%$ to $10 \%$ smooth muscle cells. ${ }^{28}$ In the cervix, collagen fibrils are 53 to $58 \pm 3 \mathrm{~nm}$ in diameter, average about 2100 to $2200 \pm 200 \mathrm{~nm}$ in length, and are packed in bundles. ${ }^{29}$ During pregnancy, the total collagen content of the cervix increases, whereas the concentration of collagen decreases. ${ }^{29-32}$ As the cervix ripens, the collagen fibrils become disorganized, and more space is created between the fibrils; thus, the concentration of collagen decreases. ${ }^{29,30,32}$ Hydroxyproline is an amino acid unique to collagen and is used to measure the collagen content of tissues. ${ }^{33}$ Hydroxyproline provides stability to the triple-stranded helical structure of collagen by forming hydrogen bonds. ${ }^{33}$ The space in between the collagen fibrils is occupied by hyaluronan, decorin, water, and enzymes, which act synergistically to ripen the cervix in preparation for labor. ${ }^{34}$ The hyaluronan content of the cervix increases with cervical ripening, creating a cell-free space, which allows substances and cells (fibroblasts, white cells, and smooth muscle cells) to move, divide, and migrate in the extracellular matrix. ${ }^{35}$ Hyaluronan has a high capacity to bind water molecules, leading to loosening collagen fibrils and softening elastin. ${ }^{36,37}$ Decorin is a proteoglycan that binds to the collagen fibrils regulating the interfibrillary distance, and increased expression results in collagen disorganization. ${ }^{32}$ Thus, this study's hypothesis was that QUS will detect the changes in the microstructure of the rat cervix associated with ripening (collagen content and concentration). 


\section{Materials and Methods}

\section{Animals}

The cervices of Sprague Dawley rats (Harlan, Indianapolis, IN) were scanned prospectively in 13 nonpregnant virgin rats and 65 timed pregnant rats (13 each at days 15, 17, 19, 20, and 21 of pregnancy). Rats were randomized into 6 groups $(\mathrm{n}=13$ rats per group). Power analysis for SD indicated that a sample size of 13 rats in each group would detect an effect size of 0.23 with a power of 0.90 , using 1-way analysis of variance (2-tailed test) and $\alpha=.05$ (nQuery Advisor; Statistical Solutions, Saugus, MA). A small effect size was chosen for this study because group intervals between scans (1-2 days) were small, and it was judged important not to waste the animal resources on an underpowered study. The experimental protocol was approved by the Institutional Animal Care and Use Committee at both the University of Illinois at Urbana-Champaign and the University of Illinois at Chicago and satisfied all campus and National Institutes of Health rules for the humane use of laboratory animals.

\section{Procedure}

Before the rats were humanely euthanized, the experiment was prepared by placing degassed water $\left(20^{\circ} \mathrm{C}-22^{\circ} \mathrm{C}\right)$ in a scanning tank (Figure 1). Ultrasonic scanning was accomplished with a custom-fabricated $70-\mathrm{MHz}$ lithium niobate transducer (National Institutes of Health Transducer Resource Center, University of Southern California, Los Angeles, CA) and a Panametrics (Waltham, MA) 5900 pulser/receiver. The water temperature was recorded for each scan. Each rat was weighed before necropsy, and the cervix was dissected, trimmed, weighed, and then immediately sealed in plastic wrap and taped to the holder (Figure 2A) that would be placed in the tank of degassed water (Figure 2B). The cervix specimen in the holder was placed at the approximate focal length of 6.75 $\mathrm{mm}$, and fine adjustments were made with the computer-controlled micropositioning system (Daedal, Inc, Harrisburg, PA) until a maximumamplitude echo signal from the tissue was obtained on the oscilloscope. Each cervix specimen was scanned $7 \mathrm{~mm}$ longitudinally in the middle of the cervix and $5 \mathrm{~mm}$ transversely at the superior margin near the internal os of the cervix. Only 2 scans of each cervix were conducted because of the time required for data acquisition (20 minutes per scan) and concern for tissue integrity due to the time that the tissue was ex vivo. A B-mode image of each specimen was generated from the RF echoes of the longitudinal scan to ensure that the tissue was in the field's depth of focus.

A backscatter power spectrum was then calculated for each scan line by gating the time signal corresponding to the chosen ROI. The total power spectrum for the ROI was determined by averaging the power spectra from each individual scan line corresponding to the ROI. The optimal size of each ROI was 10 times the wavelength axially and 5 times the beam width laterally. ${ }^{22}$ Estimates of SD, AC, and SSF were obtained for each ROI by comparing the total power spectrum with the theoretical power spectrum. Three parameterized values (SD, AC, and SSF) for each cervix specimen were determined by averaging each of them from the processed longitudinal and transverse ROIs. The average number of ROIs per scan was 350 (range, 153-567). The size of each ROI was $400 \times 400 \mu \mathrm{m}$ ( 5 beam widths laterally by 12 pulse lengths axially). ${ }^{22}$ The number of ROIs per scan depended on the thickness of the cervix tissue. These ultrasound measures would be compared to 1 mean hydroxyproline and water value for each cervix.

The loss of energy in the ultrasound beam as ultrasound propagates through tissue, insertion loss (or attenuation), is a function of tissue properties and the frequency of ultrasound.$^{38}$ Tissuespecific attenuation is essential in calculating the

Figure 1. Experimental setup.

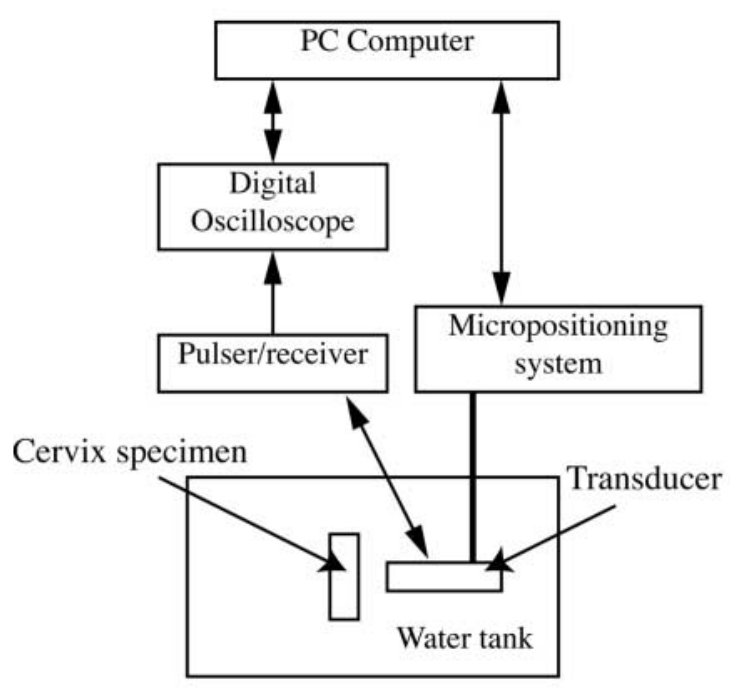




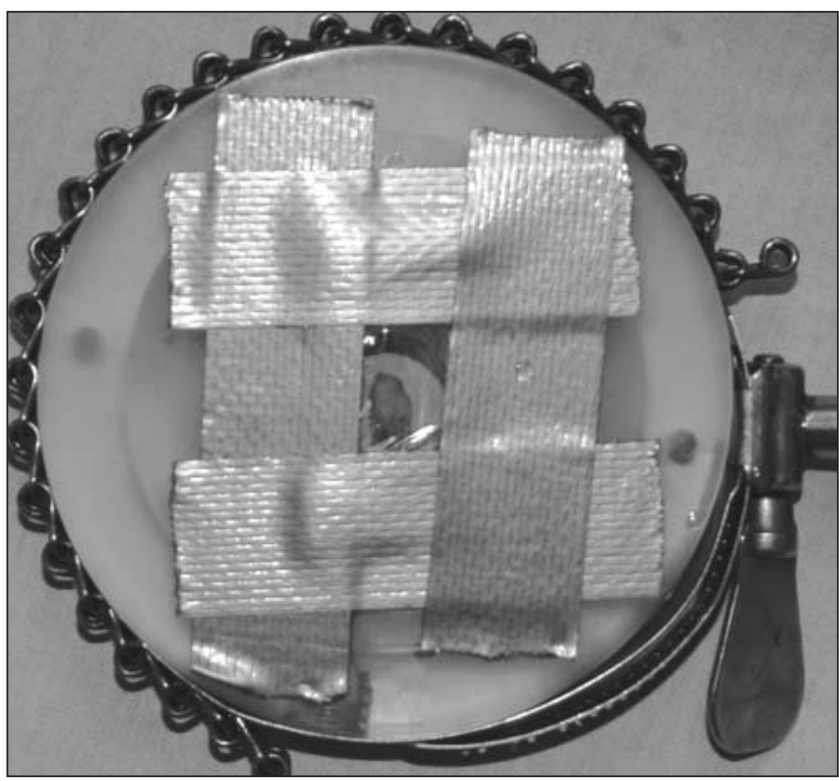

A

Figure 2. A, Cervix specimen wrapped in plastic wrap and placed in the holder. B, Cervix specimen in the holder in the scanning tank of degassed water with a $70-\mathrm{MHz}$ lithium niobate transducer placed at focal zone to the specimen $(6.5 \mathrm{~mm})$.

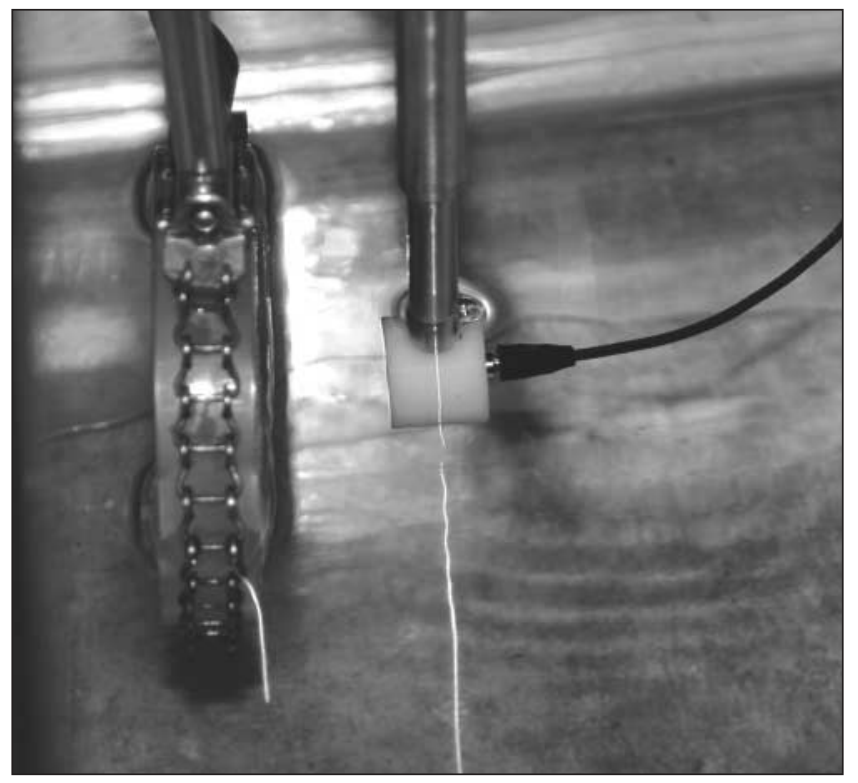

B ultrasound backscatter measures of SD, AC, and SSF. Corrections to the total backscatter power spectrum were made for tissue-specific insertion loss for each gestational age (GA) group as well as for the attenuation of the plastic wrap. Insertion loss was estimated by comparing the signal when the sample was inserted into the ultrasound beam relative to when the sample was not present. ${ }^{27}$ Each ROI was converted into a colorcoded pixel that represented the average SD and
SSF (Figure 3). All processing of ultrasound data was conducted with MATLAB software (The MathWorks, Natick, MA).

\section{Cervical Tissue Processing}

After the cervix specimen was scanned, one fifth of the cervix was dissected freehand, placed in formalin, embedded in paraffin, sectioned, mounted on a glass slide, and stained with Masson trichrome stain. To quantify the distribution of

Figure 3. Quantitative ultrasonographic images of rat 64 cervix (day 19 of pregnancy). A, Image of SD where each pixel represents 1 mean ROI in micrometers (range, 2-12 $\mu \mathrm{m}$ ). B, Image of SSF where each pixel represents 1 mean ROI in negative decibels (range, -2 to $-20 \mathrm{~dB}$ ).

A

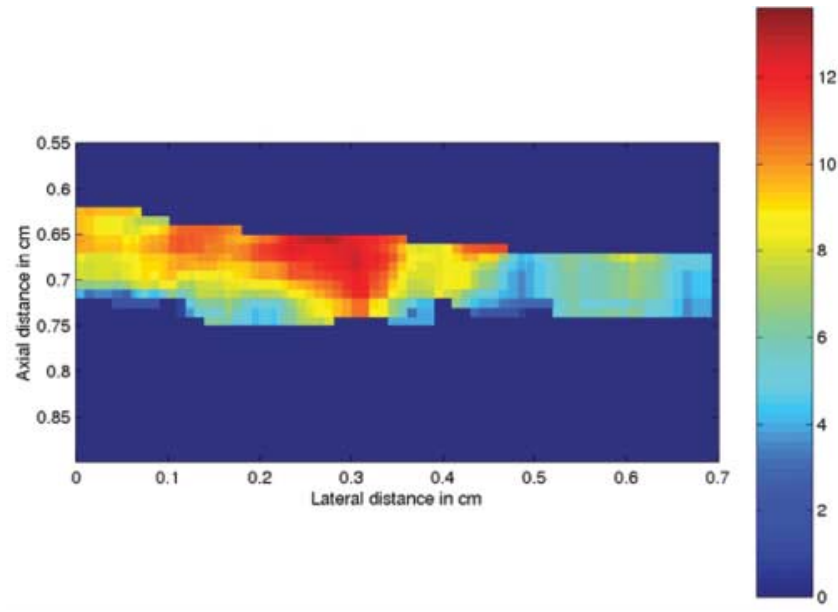

B

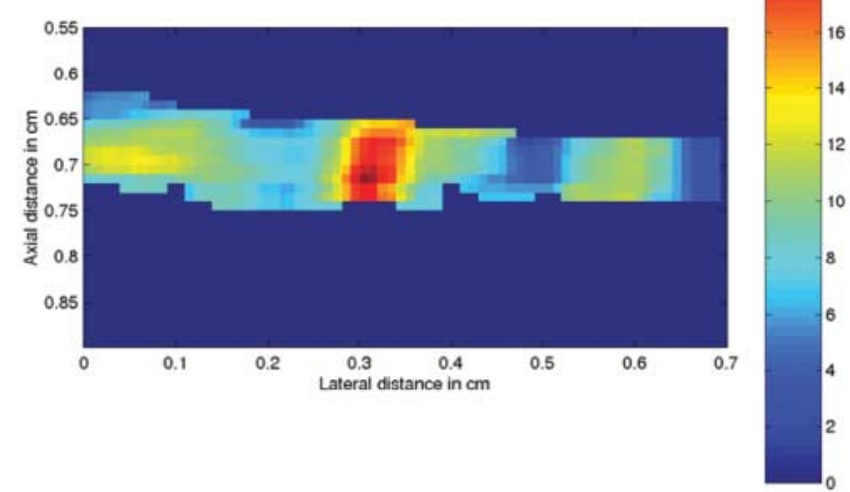


collagen, a transverse and longitudinal section of each specimen was photographed at $\times 20$ magnification using a Nikon microscope imaging workstation (Nikon Instruments Inc, Melville, $\mathrm{NY}$ ) and saved as digital images that were colorized in Photoshop (Adobe Systems Inc, San Jose, CA). The blue-stained collagen areas on the histologic slide were colorized to black, and the rest of the tissue was colorized to white (Figure 4). The black and white pixels were counted with a MATLAB program to quantify the collagen in each histologic slide.

The remainder of the specimen was flash frozen in liquid nitrogen until a solid pellet was formed, wrapped, and placed in a plastic bag in a $-70^{\circ} \mathrm{C}$ freezer until all the samples could be analyzed at the same time. Before chemical analysis at the University of Illinois at UrbanaChampaign, Department of Animal Sciences, samples were ground under liquid nitrogen. Water, nitrogen, and hydroxyproline contents of the specimens were analyzed by methods 934.01, 992.15, and 990.26, respectively, of the Official Methods of Analysis of the Association of Official Analytical Chemists. ${ }^{39}$ The nitrogen content of the cervix was measured to produce a crude protein value for each cervix. Hydroxyproline was calculated as a portion from the crude protein. To obtain water content, a subsample of each specimen was placed in previously dried crucibles, weighed, dried in a $105^{\circ} \mathrm{C}$ oven overnight, and weighed again. The percentage of dry matter was calculated by subtracting the wet weight in the crucible from the dry weight and multiplying by 100 (method 934.01 ) with an accuracy of $\pm 0.4 \mathrm{mg} .{ }^{39}$ The sensitivity to detect a difference in water percentage was $\pm 0.01 \% .^{39}$ After the water content was determined, these specimens were used to determine total nitrogen via combustion using a Leco FP2000 analyzer (Leco Corporation, St Joseph, MN) (method 992.15). Another subsample of each specimen was hydrolyzed in 6$\mathrm{N}$ hydrochloric acid for 16 hours in a $105^{\circ} \mathrm{C}$ oven; water was added to the test tube and mixed; and the aliquot was filtered through Whatman 541 filter paper (Whatman Inc, Florham Park, NJ) with activated charcoal (method 990.26). ${ }^{39}$ The filtrate aliquot was then diluted by a factor of 10 , and $2 \mathrm{~mL}$ of chloramine $\mathrm{T}$ (oxidant solution) was added to each tube. After 20 minutes, $1 \mathrm{~mL}$ of 4-dimethylaminobenzaldehyde (color reagent) was added to each tube, and the tubes were incubated for $15 \mathrm{~min}$ utes in a $60^{\circ} \mathrm{C}$ water bath. The tubes were cooled under running water for 3 minutes and then read with a spectrophotometer at 558 $\mathrm{nm} .{ }^{39}$ The accuracy of the method to detect a difference in hydroxyproline was a hydroxyproline concentration of 1.2 to $4.8 \mu \mathrm{g} / \mathrm{mL}$. The laboratory conducting the compositional analyses was blinded to the GA group of the specimens.

Figure 4. A, Masson trichrome-stained histologic slide (original magnification $\times 20$ ) of a longitudinal section of the cervix of rat 64 (day 19 of pregnancy). Collagen is stained blue, and smooth muscle is pink/red. The solid bar represents $100 \mu \mathrm{m}$. B, Colorized histologic slide. Collagen was colorized black, and the rest of the tissue was white. The ratio of collagen pixels to white pixels (averaging cross and longitudinal sections) was 0.45 .

A

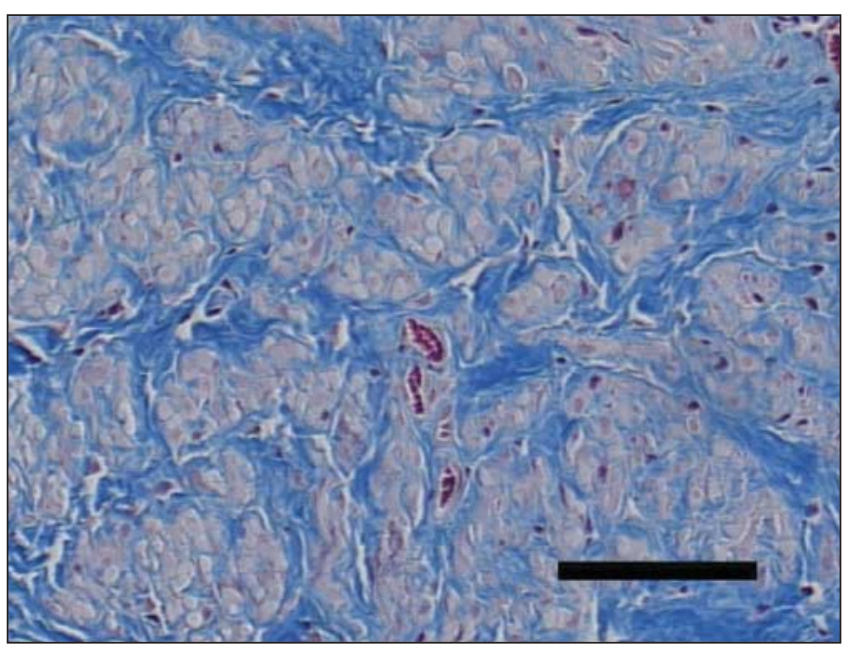

B

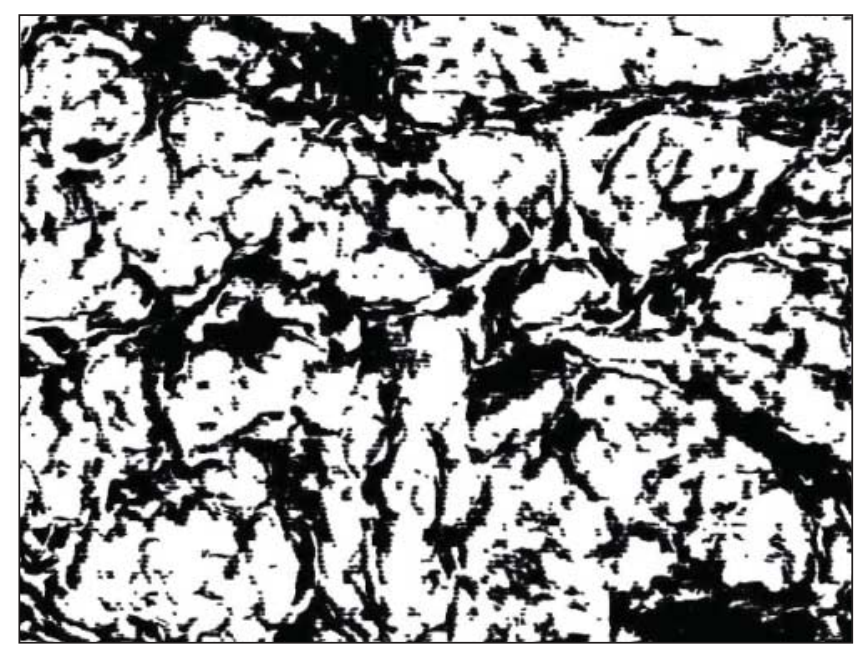




\section{Statistical Analysis}

The data were analyzed with SPSS 13.0 software (SPSS Inc, Chicago, IL), tested for normality, linearity, and outliers. Descriptive statistics were calculated for all the variables and analyzed by group. One-way analysis of variance (F ratio, between-groups degrees of freedom, followed by within-groups degrees of freedom) with Tukey post hoc tests (to determine between group differences) were conducted for age of the rats, duration of the experiment, pre-experiment and post-experiment cervix weight, percentage of water/cervix, percentage of hydroxyproline/ cervix, SD, AC, and SSF to determine significant differences by GA group. Pearson correlations ( $r$ statistic: + for a positive relationship and - for a negative relationship) were conducted to determine the relationship between the following variables: GA group, hydroxyproline, SD, AC, SSF, and collagen-stained pixels. Discriminant analysis was used to predict GA group from the ultrasound variables SD, SSF, and AC, as well as to build a statistical prediction model for future use. Discriminant analysis was used rather than regression analysis because of the colinearity of SD, AC, and SSF with each other. Colinearity would violate an important assumption of independence of the variables for regression analysis. ${ }^{40}$ The level for statistical significance was $P<.05$.

\section{Results}

Complete data were collected on 78 Sprague Dawley rats, including 65 timed pregnant rats and 13 virgin nonpregnant Sprague Dawley rats. The 13 nonpregnant rats were a group of rats that were scheduled to be euthanized from another investigator's study and thus were considerably older than the timed pregnant rats purchased specifically for this study. In addition to data being acquired for all 78 rats, insertion loss data were collected on 3 rats in each group for the last 18 rats in the study. Table 1 lists rat and pup characteristics.

For each sample, the mean SD, AC, and SSF values were determined. Figure 3 shows that for 2 of these parameters, there was heterogeneity as a function of position. Table 2 lists the ultrasound and compositional measures of the cervix by group. Except for the day 17 group, which had the largest SD, there was a trend of increasing SD from the day 15 group toward day 21 of pregnan- cy (Table 2). Except for the day 17 group, which had the least AC, the AC decreased from the nonpregnant group to the day 21 group. Scatterer strength factor was the greatest in the nonpregnant group and the least in the day 21 group. Day 17 SSF was almost as low as the day 21 SSF. There was a significant linear decrease in the percentage of hydroxyproline $(P<.0001)$ and an increase in the micrograms of hydroxyproline per cervix $(P<.003)$ from the day 15 group to the day 21 group (Table 2). These findings suggest that, as pregnancy progresses, the total hydroxyproline content per cervix increases, whereas the concentration of hydroxyproline decreases.

The percentage of water per cervix $(P=.24)$ and the ratio of collagen-stained pixels on the histologic slides $(P=.17)$ were not significantly different among the groups (Table 2). The percentage of hydroxyproline was significantly correlated with group $(r=-0.70 ; P<.001)$. The ratio of collagen-stained pixels on the histologic slides was significantly correlated with AC $(r=+0.39)$ and SSF $(r=+0.35 ; P<.001)$.

To predict the day of gestation, and thus cervical ripening, from the ultrasound variables, discriminant analysis was used. Two discriminant analysis models were calculated to predict group classification: 1 that included the nonpregnant group with the pregnant groups and 1 that only included the pregnant groups.

The first discriminant analysis model, which included the nonpregnant and pregnant groups, determined that SD, AC, and SSF significantly predicted group assignment (Wilks $\lambda=0.187$; $\chi^{2}[15]=121.6 ; P<.0001$ ), with $70 \%$ of the variance explained by the model. Scatter strength factor contributed most to the model $(r=+0.98)$, followed by AC $(r=+0.73)$ and SD $(r=+0.23)$. With all the ultrasound variables entered into the model, the following discriminant function equation was calculated to predict GA group: $\mathrm{GA}=1.20 \times \mathrm{SSF}-0.28 \times \mathrm{AC}+0.16 \times \mathrm{SD}$. Overall, this model correctly predicted group membership for $56.4 \%$ of the cases (Figure 5A). Predicting the exact day of gestation is difficult in any biological model. Therefore, the model was analyzed within 2 days of the predicted gestational day as well (Figure 5A). The number of correct gestational day classifications increased from $56.4 \%$ in the predicted model to $77 \%$ within 2 days of prediction analysis. The model predicted $100 \%$ of the nonpregnant group and performed the poorest in the day 21 group. 
Table 1. Characteristics of the Sample by Groups

\begin{tabular}{lcccccccc}
\hline Characteristic & $\mathbf{n}$ & Nonpregnant & Day 15 & Day 17 & Day 19 & Day 20 & Day 21 & Statistics \\
\hline Rat age, $\mathrm{d}^{*}$ & 78 & $215(25)$ & $108(14)$ & $108(14)$ & $108(14)$ & $110(13)$ & $111(11)$ & $\mathrm{F}_{5,72}=93.61 ; P<.0001$ \\
Rat weight, g & 78 & $301(17)$ & $286(15)$ & $298(16)$ & $323(39)$ & $349(24)$ & $353(36)$ & $\mathrm{F}_{5,72}=14.86 ; P<.0001$ \\
No. of pups & 65 & 0 & 13 & 12 & 11 & 13 & 13 & $\mathrm{~F}_{4,60}=0.84 ; P=.51$ \\
Pup weight, g & 65 & 0 & $0.4(0.1)$ & $1.1(0.2)$ & $2.7(0.4)$ & $4.2(0.3)$ & $5.4(0.6)$ & $\mathrm{F}_{4,60}=5.26 ; P<.0001$ \\
Duration of experiment, mint & 78 & $99(51)$ & $90(10)$ & $95(33)$ & $108(53)$ & $88(32)$ & $105(37)$ & $\mathrm{F}_{5,72}=0.50 ; P=.77$ \\
Rat cervix weight, mg & 78 & $66(13)$ & $74(10)$ & $88(11)$ & $97(11)$ & $99(18)$ & $114(23)$ & $\mathrm{F}_{5,72}=17.33 ; P<.0001$ \\
Cervix length, mm & 78 & $5.9(0.6)$ & $6.4(0.5)$ & $6.7(0.6)$ & $6.8(0.8)$ & $5.8(0.8)$ & $5.9(0.7)$ & $\mathrm{F}_{5,72}=5.26 ; P<.0001$ \\
Cervix width, mm & 78 & $3.0(0.4)$ & $3.1(0.5)$ & $3.4(0.4)$ & $3.3(0.6)$ & $3.3(0.5)$ & $3.7(0.6)$ & $F_{5,72}=3.28 ; P=.01$ \\
Cervix depth, mm & 78 & $4.4(0.4)$ & $4.7(0.5)$ & $4.7(0.7)$ & $4.7(0.7)$ & $5.1(0.4)$ & $6.1(1.1)$ & $F_{5,72}=9.82 ; P<.0001$ \\
\hline
\end{tabular}

Values are expressed as mean (standard deviation); $n=13$ rats in each group.

${ }^{*}$ Age was not significantly different among pregnant groups; $\mathrm{F}_{4,60}=0.14 ; P=.96$.

†Time from euthanasia until the cervix specimen was frozen.

Because the nonpregnant group SD, AC, and SSF were so different from those of the pregnant groups, a second discriminant analysis was conducted considering only the pregnant groups in the model. The model was again found to be significant (Wilks $\lambda=0.451 ; \chi^{2}[12]=47.84 ; P<.0001$ ), with $86 \%$ of the variance explained by the model. In this model, SD contributed most to the model $(r=+0.96)$, followed by AC $(r=+0.75)$ and SSF $(r=-0.30)$. The following discriminant function equation was calculated to predict GA group: $\mathrm{GA}=1.26 \times \mathrm{SD}-0.787 \times \mathrm{SSF}+0.594 \times$ AC. Again, most of the errors in classification were within 2 days of the predicted group. Correct classification of groups increased from $46 \%$ in the predicted model to $69 \%$ within 2 days of the predicted model (Figure 5B). The model predicted the day 15 and 17 groups the best and the day 21 group the least.
Insertion loss was the greatest for the nonpregnant group and the least for the day 21 group across the frequency range between 30 and 70 $\mathrm{MHz}$ (Figure 6). Insertion loss for the water path of the ultrasound beam was given as a reference point.

\section{Discussion}

In this study, QUS was used to examine the microstructural changes in the cervix throughout pregnancy. Based on previous work, ${ }^{24,25,27}$ it was hypothesized that ultrasound scattering was related to the organization and content of collagen in tissues. As pregnancy progressed, the total collagen content of the cervix increased, whereas the concentration decreased as fibers disorganized and more space was created between the collagen fibers..$^{32}$ In a study of cervical ripening in

Table 2. Sonographic and Compositional Measures of the Cervix

\begin{tabular}{|c|c|c|c|c|c|c|c|c|}
\hline GA of Rat & $\mathbf{n}$ & $\begin{array}{l}S_{w_{*}} \\
\mu m^{*}\end{array}$ & $\begin{array}{c}\mathrm{AC} \\
\mathrm{dB} / \mathrm{mm}^{3^{\dagger}}\end{array}$ & $\begin{array}{c}\mathrm{SSF}, \\
\mathrm{dB} / \mathrm{mm}^{3^{*}}\end{array}$ & $\begin{array}{l}\text { Hydroxyproline/ } \\
\text { Cervix, } \%{ }^{\S}\end{array}$ & $\begin{array}{l}\text { Hydroxyproline/ } \\
\text { Cervix, } \mu \mathrm{g} / \mathrm{mg}\end{array}$ & $\begin{array}{l}\text { Water/ } \\
\text { Cervix, \% }\end{array}$ & $\begin{array}{c}\text { Ratio of } \\
\text { Collagen Pixels" }\end{array}$ \\
\hline Nonpregnant & 13 & $10.4(0.6)^{\#}$ & $-118(6)^{\# * \star}$ & $2.8(1)^{\# * *}$ & $0.88(0.1)^{\#^{* *}}$ & $568(75)^{\# \star \star}$ & $68(5)$ & $0.83(0.3)$ \\
\hline Day 15 & 13 & $8.2(1.3)^{\# * *}$ & $-130(7)^{\star \star}$ & $-1.5(1)$ & $0.72(0.1)^{\# * \star}$ & $532(83)^{\star *}$ & $69(8)$ & $0.69(0.3)$ \\
\hline Day 17 & 13 & $11.2(0.8)^{\#}$ & $-144(7)^{\#}$ & $-2.3(2)$ & $0.68(0.1)$ & $603(103)$ & $68(5)$ & $0.61(0.2)$ \\
\hline Day 19 & 13 & $8.6(1.2)$ & $-130(5)^{\star *}$ & $-1.2(1)$ & $0.62(0.1)^{\#}$ & $605(75)^{\#}$ & $69(6)$ & $0.69(0.2)$ \\
\hline Day 20 & 13 & $8.8(1.0)$ & $-136(8)$ & $-2.2(2)$ & $0.64(0.1)^{\#}$ & $640(131)^{\#}$ & $69(4)$ & $0.58(0.2)$ \\
\hline Day 21 & 13 & $10.0(1.7)^{\#}$ & $-141(11)^{\#}$ & $-2.7(2)$ & $0.62(0.1)^{\#}$ & $707(153)^{\#}$ & $68(6)$ & $0.70(0.3)$ \\
\hline Statistic & & $\begin{array}{c}F_{5,72}=10.72 \\
P<.0001\end{array}$ & $\begin{array}{c}F_{5,72}=19.47 \\
P<.0001\end{array}$ & $\begin{array}{c}F_{5,72}=26.47 \\
P<.0001\end{array}$ & $\begin{aligned} F_{5,72} & =107.45 \\
P & <.0001\end{aligned}$ & $\begin{array}{c}\mathrm{F}_{5,72}=4.09 \\
P<.003\end{array}$ & $\begin{aligned} \mathrm{F}_{5,72} & =0.24 \\
P & =.24\end{aligned}$ & $\begin{array}{c}F_{5,72}=1.6 \\
P=.17\end{array}$ \\
\hline
\end{tabular}

Values are expressed as mean (standard deviation)

*Scatterer diameter = correlational length of the scatterer; differences among groups.

${ }^{\dagger}$ Acoustic concentration $=$ number of scatterers $/ \mathrm{mm}^{3}$ - (average impedance of scatterers) ${ }^{2}$.

${ }^{\ddagger}$ Scatterer Strength Factor $=\mathrm{AC} \times$ (average radius of scatterers) ${ }^{6}$.

${ }^{\S}$ Concentration of hydroxyproline per cervix, wet weight.

"Content of hydroxyproline per total cervix, wet weight.

"Ratio of collagen-stained pixels compared with stained cells (smooth muscles and white cells) per digital histologic slide at $\times 20$ magnification.

\#** Tukey post hoc tests to determine between-group differences: ${ }^{\#} P<.01$ versus day 15 group; ${ }^{* *} P<.01$ versus day 21 group. 


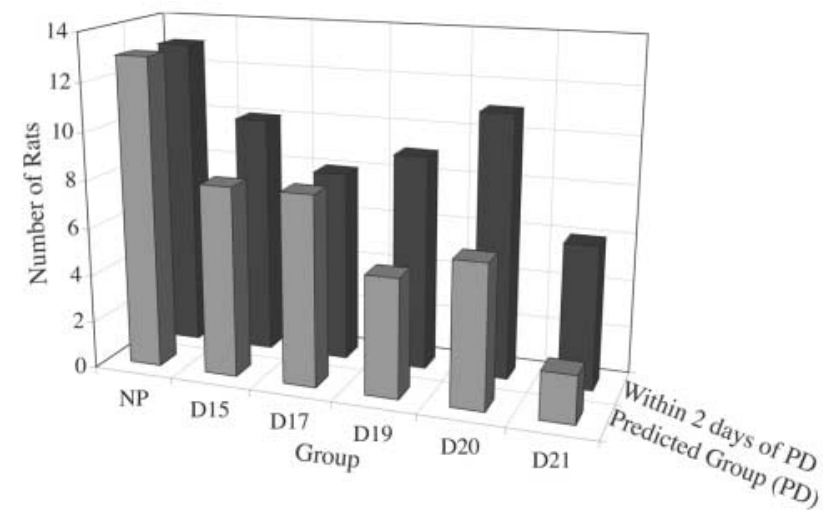

A

Figure 5. A, Discriminant analysis group classification for the nonpregnant (NP) and pregnant groups, using the ultrasound measures of SD, AC, and SSF for the predicted group (Wilks $\lambda=0.187 ; \chi^{2}[15]=121.6 ; P<.0001$ ) and within 2 days of the predicted group; $n=13$ per group. B, Discriminant analysis group classification for the pregnant groups only, using the ultrasound measures of SD, AC, and SSF for the predicted group (Wilks $\lambda=0.451$; $\chi^{2}[12]=47.8 ; P<.0001$ ) and within 2 days of the predicted group; $n=13$ per group. $D$ indicates day of pregnancy; and PD, predicted day of gestation.

pregnant rats, Feltovich and coworkers ${ }^{29}$ found little change in the diameter of the collagen fibrils (53 $\pm 3 \mathrm{~nm}$ control, $58 \pm 3 \mathrm{~nm}$ after ripening) but significantly more space between fibrils as the cervix ripened. In our study, SD varied little during pregnancy, whereas AC decreased significantly as pregnancy progressed. This suggests that the scattering size did not markedly change, but the concentration of the scatterers in the cervix tissue did decrease as pregnancy progressed. There was a significant correlation between the ratio of col-

Figure 6. Mean insertion loss in decibels for cervix samples by groups (temperature, $21.8^{\circ} \mathrm{C}$ ) compared with insertion loss of water (temperature, $22^{\circ} \mathrm{C}$ ) for frequency ranges of 30 to $70 \mathrm{MHz} ; \mathrm{n}=3$ per group. $\mathrm{D}$ indicates day of pregnancy; and NP, nonpregnant.

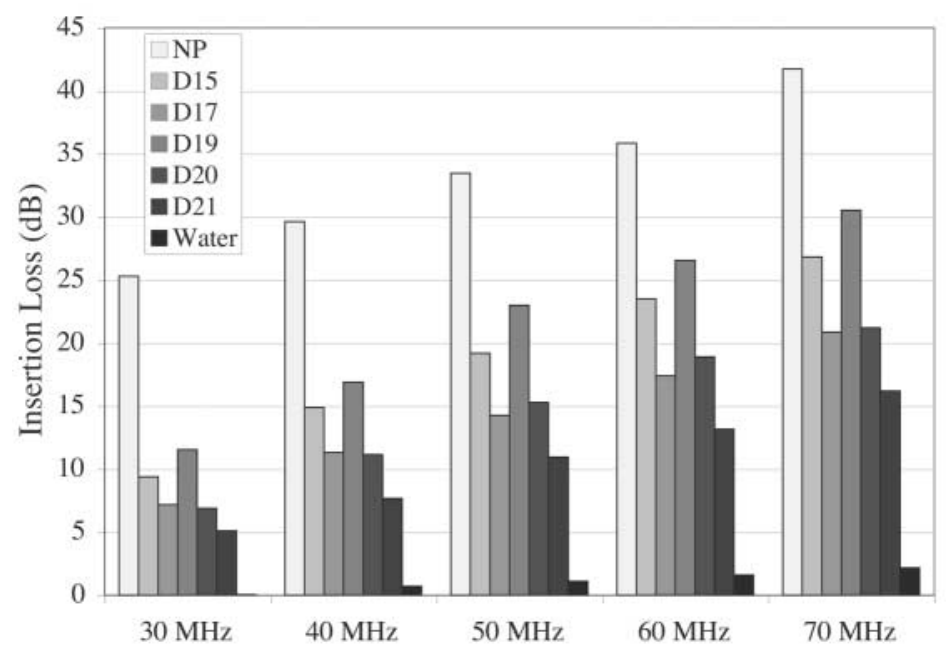

lagen pixels on the colorized histologic slides and AC and SSF, giving further evidence to suggest a relationship between scattering and collagen. Future research is warranted to relate interfibrillary distance of collagen fibrils to SD, AC, and SSF. However, our study did determine significant correlations between the hydroxyproline percentage and content of the cervix specimens with GA group, AC, and SSF.

The day 17 group presented a different pattern for SD, AC, and SSF. The data were analyzed for outliers, and the standard deviations were very similar to those of the other groups. The differences between the day 17 group and the other groups cannot be explained by differences in methods. Around day 17 or 18 in the rat pregnancy, marked changes occurred with collagen content and glycosaminoglycan content and distribution in the ripening cervix. ${ }^{30,32}$ It is possible that the day 17 pattern of SD, AC, and SSF showed these morphologic changes, heralding the onset of cervical ripening.

The cervical water content did not significantly vary among groups. All animals were allowed food and water ad libitum before scanning. A previous report ${ }^{30}$ noted only a $3 \%$ increase in cervix water content from the nonpregnant state until term, and proteoglycans have been found to be the main component in the extracellular matrix during ripening. ${ }^{34}$

The marked GA differences in insertion loss of the cervix were unexpected findings. Tissuespecific attenuation is essential in calculating the 
ultrasound backscatter measures of SD, AC, and SSF. Insertion loss in tissues has been related to the structure of tissue ${ }^{41}$ and the type of tissue.$^{25}$ In tissues such as the cervix, where dynamic structural changes occur with cervical ripening, it is important to use a measured insertion loss rather than a value that is a tissue average. The nonpregnant cervix had the highest insertion loss, and the day 21 pregnant cervix had the least. In this study, insertion loss was only measured in 3 rats in each group to obtain a mean attenuation coefficient for each GA group. Refining the methods by measuring insertion loss for each animal and using that unique value in the calculation of QUS may improve detection of cervical ripening. In summary, this article reports the use of QUS to detect GA changes in the cervix. Although the discriminant function analysis significantly predicted GA group using the ultrasound parameters of SD, AC, and SSF, for this technology to be useful in humans, it must predict the later days of pregnancy with more precision. We anticipate that we can improve the prediction of cervical ripening with QUS by (1) making the measurements in vivo; (2) measuring insertion loss in each individual case; and (3) using the unique insertion loss measurement for each case to calculate SD, AC, and SSF. Before this technology can be used with humans, the following must occur: (1) the frequency that best shows the microstructural changes in the cervix associated with cervical ripening must be determined; (2) the content and distribution of collagen from histologic slides must be validated with QUS; and (3) an in vivo scanning method must be developed in the animal model. The computer processing of QUS could be automated, thus making it user-friendly and fast. Quantitative ultrasound could be an added feature to commercial ultrasound systems available to clinicians. This technology has the potential to affect the care of pregnant women by providing a noninvasive technique to detect cervical ripening.

\section{References}

1. Martin JA, Hamilton BE, Sutton PD, Ventura SJ, Menacker F, Munson ML. Births: final data for 2003. Natl Vital Stat Rep 2005; 54:1-116.

2. Lorenz JM. The outcome of extreme prematurity. Semin Perinatol 2001; 25:348-359.

3. St John EB, Nelson KG, Cliver SP, Bishnoi RR, Goldenberg $\mathrm{RL}$. Cost of neonatal care according to gestational age at birth and survival status. Am J Obstet Gynecol 2000; 182:170-175.

4. Hamilton BE, Ventura SJ, Martin JA, Sutton PD. Preliminary births for 2004. In: Health E-Stats. Hyattsville, MD: National Center for Health Statistics; 2005. Available at: http:// www.cdc.gov/nchs/products/pubs/pubd/hestats/prelim_bir ths/prelim_births04.htm.

5. Office of Disease Prevention and Health Promotion, Office of Public Health and Science, US Department of Health and Human Services. Healthy People 2010: Understanding and Improving Health. Washington, DC: US Government Printing Office; 2000.

6. Danforth DN. The fibrous nature of the human cervix and its relation to the isthmic segment in gravid and nongravid uteri. Am J Obstet Gynecol 1947; 53:541-557.

7. Chwalisz K, Garfield RE. Role of nitric oxide in the uterus and cervix: implications for the management of labor. J Perinat Med 1998; 26:448-457.

8. Mahendroo MS, Porter A, Russell DW, Word RA. The parturition defect in steroid 5alpha-reductase type 1 knockout mice is due to impaired cervical ripening. Mol Endocrinol 1999; 13:981-992.

9. Word RA, Landrum CP, Timmons BC, Young SG, Mahendroo MS. Transgene insertion on mouse chromosome 6 impairs function of the uterine cervix and causes failure of parturition. Biol Reprod 2005; 73:1046-1056.

10. Kremkau FW. Diagnostic Ultrasound: Principles and Instruments. Philadelphia, PA: WB Saunders Co; 1998.

11. Shung KK, Thieme GA. Biologic tissues as ultrasonic scattering media. In: Shung KK, Thieme GA (eds). Ultrasonic Scattering in Biologic Tissues. Boca Raton, FL: CRC Press; 1993:53-74.

12. Oelze ML, Zachary JF, O'Brien WD Jr. Characterization of tissue microstructure using ultrasonic backscatter: theory and technique for optimization using a Gaussian form factor. J Acoust Soc Am 2002; 112:1202-1211.

13. Insana MF. Modeling acoustic backscatter from kidney microstructure using an anisotropic correlation function. J Acoust Soc Am 1995; 97:649-655.

14. Oelze ML, O'Brien WD Jr. Frequency-dependent attenuation-compensation functions for ultrasonic signals backscattered from random media. J Acoust Soc Am 2002; 111:2308-2319.

15. Insana MF, Brown DG. Acoustic scattering theory applied to soft biological tissues. In: Shung KK, Thieme GA (eds). Ultrasonic Scattering in Biologic Tissues. Boca Raton, FL: CRC Press; 1993:75-124.

16. Faran JJ. Sound scattering by solid cylinders and spheres. J Acoust Soc Am 1951; 23:405-418.

17. Insana MF, Hall TJ. Parametric ultrasound imaging from backscatter coefficient measurements: image formation and interpretation. Ultrason Imaging 1990; 12:245-267.

18. Lizzi FL, Astor M, Kalisz A, et al. Ultrasonic spectrum analysis for assays of different scatterer morphologies: theory 
and very-high frequency clinical results. Int J Imaging Syst Technol 1997; 8:3-10.

19. Lizzi FL, Ostromogilsky M, Feleppa EJ, Rorke MC, Yaremko MM. Relationship of ultrasonic spectral parameters to features of tissue microstructure. IEEE Trans Ultrason Ferroelectr Freq Control 1986; 33:319-329.

20. Nassiri DK, Hill CR. The use of angular acoustic scattering measurements to estimate structural parameters of human and animal tissues. J Acoust Soc Am 1986; 79:2048-2054.

21. Nicholas D, Hill CR, Nassiri DK. Evaluation of backscattering coefficients for excised human tissues: principles and techniques. Ultrasound Med Biol 1982; 8:7-15.

22. Oelze ML, O'Brien WD Jr. Defining optimal axial and lateral resolution for estimating scatterer properties from volumes using ultrasound backscatter. J Acoust Soc Am 2004; 115:3226-3234.

23. Oelze ML, Zachary JF, O'Brien WD Jr. Parametric imaging of rat mammary tumors in vivo for the purposes of tissue characterization. J Ultrasound Med 2002; 21:1201-1210.

24. Fields S, Dunn F. Correlation of echographic visualizability of tissue with biological composition and physiological state. J Acoust Soc Am 1973; 54:809-812.

25. Hall CS, Nguyen CT, Scott MJ, Lanza GM, Wickline SA. Delineation of the extracellular determinants of ultrasonic scattering from elastic arteries. Ultrasound Med Biol 2000; 26:613-620.

26. Pohlhammer J, O'Brien WD Jr. Dependence of the ultrasonic scatter coefficient on collagen concentration in mammalian tissues. J Acoust Soc Am 1981; 69:283-285.

27. Hall CS, Scott MJ, Lanza GM, Miller JG, Wickline SA. The extracellular matrix is an important source of ultrasound backscatter from myocardium. J Acoust Soc Am 2000; 107:612-619.

28. Winkler $\mathrm{M}$, Rath $\mathrm{W}$. Changes in the cervical extracellular matrix during pregnancy and parturition. J Perinat Med 1999; 27:45-60.

29. Feltovich $\mathrm{H}$, Ji H, Janowski JW, Delance NC, Moran CC, Chien EK. Effects of selective and nonselective PGE2 receptor agonists on cervical tensile strength and collagen organization and microstructure in the pregnant rat at term. Am J Obstet Gynecol 2005; 192:753-760.

30. Goilichkowski AM, King SR, Mascaro K. Pregnancy related changes in rat cervix glycosaminogylcans. Biochem J 1980; 192:1-8.

31. Leppert PC. Anatomy and physiology of cervical ripening Clin Obstet Gynecol 1995; 38:267-279.

32. Leppert PC, Kokenysei R, Klemenich CA, Fisher J. Further evidence of a decorin-collagen interaction in the disruption of cervical collagen fibers during rat gestation. Am J Obstet Gynecol 2000; 182:805-811.

33. Alberts B, Johnson A, Lewis J, Raff M, Roberts K, Walter P. Molecular Biology of the Cell. New York, NY: Garland Science; 2002.
34. Buhimschi IA, Dussably L, Buhimschi CS, Ahmed A, Weiner CP. Physical and biomechanical characteristics of rat cervical ripening are not consistent with increased collagenase activity. Am J Obstet Gynecol 2004; 191:1695-1704.

35. Toole BP. Hyaluronan in morphogenesis. Semin Cell Dev Biol 2001; 12:79-87.

36. Obara $\mathrm{M}$, Hirano $\mathrm{H}$, Ogawa $\mathrm{M}$, et al. Changes in molecular weight of hyaluronan and hyaluronidase activity in uterine cervical mucus in cervical ripening. Acta Obstet Gynecol Scand 2001; 80:492-496.

37. Uchiyama T, Matsumoto T, Suzuki $Y$, Ishida M, Obara T, Kanayama T. Endogenous hyaluronan: a cytokine-like factor present in rabbit uterine cervix during pregnancy. Biol Pharm Bull 2004; 27:1907-1912.

38. Kossoff G. Basic physics and imaging characteristics of ultrasound. World J Surg 2000; 24:134-142.

39. Horwitz W. Official Methods of Analysis of the Association of Official Analytical Chemists. Gaithersburg, MD: Association of Official Analytical Chemists; 2002.

40. Pedhazur EJ. Multiple Regression in Behavioral Research: Explanation and Prediction. London, England: Thomson Learning; 1997.

41. Njeh CF, Fuerst T, Diessel E, Genant HK. Is quantitative ultrasound dependent on bone structure? A reflection. Osteoporosis Int 2001; 12:1-15. 